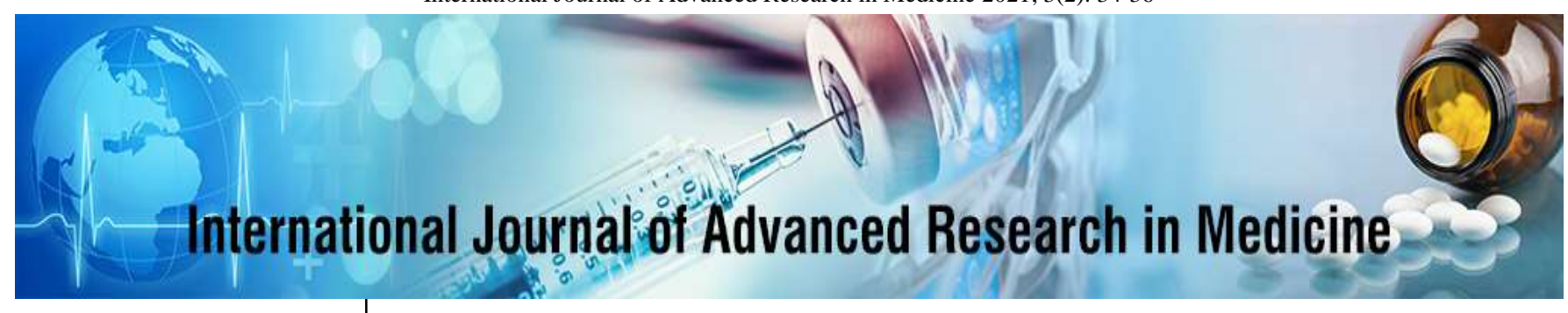

E-ISSN: 2706-9575

P-ISSN: 2706-9567

IJARM 2021; 3(2): 34-36

Received: 19-05-2021

Accepted: 24-06-2021

Andreas Fotios Tsimerakis Department of Physiotherapy, Faculty of Health Sciences

International Hellenic

University - Alexander

Campus, Sindos, Thessaloniki, Greece

\section{Anastasios Kottaras}

Department of Physiotherapy,

Faculty of Health Sciences

International Hellenic

University - Alexander

Campus, Sindos, Thessaloniki,

Greece

\section{Paris Iakovidis}

Department of Physiotherapy,

Faculty of Health Sciences

International Hellenic

University - Alexander

Campus, Sindos, Thessaloniki,

Greece

\section{Dimitrios Lytras}

Department of Physiotherapy, Faculty of Health Sciences

International Hellenic

University - Alexander

Campus, Sindos, Thessaloniki, Greece

Ioanna P Chatziprodromidou Department of Physiotherapy, Faculty of Health Sciences International Hellenic University - Alexander Campus, Sindos, Thessaloniki, Greece

Konstantinos G Papanikolaou Department of Physical Education and Sports Sciences, Democritus University of Thrace, 7th km KomotiniXanthi, Komotini, Greece

Corresponding Author: Andreas Fotios Tsimerakis Department of Physiotherapy, Faculty of Health Sciences International Hellenic University - Alexander Campus, Sindos, Thessaloniki, Greece

\title{
The effect of kinesio taping on the trunk and lower limb function in children with cerebral palsy
}

\begin{abstract}
Andreas Fotios Tsimerakis, Anastasios Kottaras, Paris Iakovidis, Dimitrios Lytras, Ioanna P Chatziprodromidou and Konstantinos G Papanikolaou
\end{abstract}

DOI: https://doi.org/10.22271/27069567.2021.v3.i2a.214

\begin{abstract}
In cerebral palsy $(\mathrm{CP})$, severe dysfunction of the posture and voluntary movement is caused, due to some damage to the brain during childhood, before it is even completely formed. For this reason, the physiotherapy approach is a demanding and time-consuming affair. The application of Kinesio Taping (KT), in combination with other physiotherapy interventions, helps in the function of the trunk and lower limbs in children with CP. The aim of this review of the literature is through recent studies to outline the effect of KT application on the function of the trunk and lower limbs in children with CP. The Google Scholar and PubMed databases were searched in English with the following keywords: Efficacy, Kinesio taping, gait, trunk, posture, cerebral palsy. The review included randomized clinical trials and pilot studies. Of the 10 articles that were included in this review, seven were randomized clinical trials and three were pilot studies. Discussion-Conclusions: The results show that the application of KT in children with $\mathrm{CP}$ has positive results in terms of increasing the function of the trunk and lower limbs. In most cases, in fact, it seemed that this happens not immediately after application, but shortly after its removal. Additionally, the combination of KT together with other physiotherapy interventions leads to even better results. More studies will be needed to further investigate its efficacy.
\end{abstract}

Keywords: Efficacy, kinesio taping, gait, trunk, posture, cerebral palsy

\section{Introduction}

Cerebral palsy (CP) is a non-evolving brain damage that occurs in childhood and to a large extent results in severe disability ${ }^{[1]}$. Spastic diplegia is more common and the lower limbs are more affected than the upper limbs ${ }^{[2]}$. It affects posture and movement, due to a disturbance or damage to the brain before it even matures or is completely formed ${ }^{[3]}$. Despite its static nature, its diversity and its clinical image that differs during the development process of the child, makes the therapeutic approach a demanding and time-consuming case for the physiotherapist ${ }^{[1]}$.

Epidemiological data show that two to three children in 1000 births are born with CP and the largest percentage concerns children who are underweight. Regarding categorization, 35\% are hemiplegic, $57 \%$ quadriplegic and $28 \%$ diplegic. For example, in the USA the incidence is from $1.4 \%$ to $2.3 \%$ with spastic diplegia having increasing tendencies (32\%) with a simultaneous decline of the athetoid type of $\mathrm{CP}^{[4]}$.

Physiotherapy can make a significant contribution to the overall improvement of functioning in children with $\mathrm{CP}$, as it facilitates and promotes their need to participate in activities, reducing the impact of symptoms from serious injuries ${ }^{[5]}$. Such interventions aim to improve posture in order to keep the head in the middle position, to improve or avoid additional changes that will burden the structure and posture of the body, so that these people can be facilitated in achieving their daily routine and ultimately develop their autonomy ${ }^{[4]}$. For these reasons, physiotherapists use a wide variety of interventions that promote voluntary mobility ${ }^{[5]}$, especially in cases of spasticity, such as those presented in this review, in which the disorders are related to the reduction of range of motion due to increased muscle tone ${ }^{[6]}$. One of these interventions is the application of Kinesio Tape (KT), which the present literature review is focused on.

The purpose of this review of the literature is through recent studies to outline the effect of the application of KT on the function of the trunk and lower limbs in children with CP. 


\section{Literature review}

The Google Scholar and PubMed databases were searched in English with the following keywords: Efficacy, Kinesio taping, gait, trunk, posture, cerebral palsy. The review included seven randomized clinical trials and three pilot studies. The following are the main conclusions from the articles included in this review.

Tabatabaee et al. ${ }^{[7]}$ in a sample of 30 children with $\mathrm{CP}$, studied the effect of KT on range of motion, spasticity and lower limb function. Patients were divided into two equal groups of 15 and received treatment for two weeks. In the intervention group KT was applied with an appropriate tension and in the control group the application was not done in the suggested way. The results showed that there was no significant change in the first two days in either the intervention group or the control group in terms of range of motion $(p=.582)$, muscle tone $(p=.317)$ and movement function $(p=.320)$. In contrast, after two weeks there was a significant improvement in knee muscle tone for the intervention group $(p=.014)$, but not for the control group ( $p=.31$ ), making the KT application capable of reducing spasticity, increasing range of motion and improve function in children with $\mathrm{CP}$.

In their study, Jung et al. ${ }^{[8]}$ in a sample of four children with spastic diplegia, investigated the effect of KT application on gait. Participants were asked to walk for 10 meters with and without the tape and were videotaped. This was done a total of three times with an interval of five minutes each time. Various parameters, such as gait speed, step and stride length, gait rate, single-leg and bipedal support were evaluated with GAITRite. The results showed significant progress $(p<.05)$ in gait speed, single-leg support, step and stride length using KT in the right leg of the patients. No progress was seen in gait for both legs, as well as bipedal support and single-leg support for the left leg.

Furthermore, Dos Santos et al. ${ }^{[9]}$ studied the efficacy of KT on the transition from the sitting to the standing position in children with CP. Out of a total of 73 children, 11 were analyzed in which the application was made in the rectus femoris muscle of the affected limb. The intervention was performed with KT, without it and with placebo treatment. Muscle activity, trunk function and lower limb alignment were assessed. The results showed that the application of the tape increased the function of the rectus femoris, reduced the large flexion of the trunk, hip, knee and ankle and increased the trunk activity at the end of the movement from sitting to standing in relation with the other two applications.

Moreover, Partoazar et al. ${ }^{[10]}$ in a sample of 38 children with CP, studied the effect of the application of KT on the trunk, in terms of static and dynamic balance, as well as functional mobility. In a randomized distribution, individuals were divided into two equal groups. In the intervention group the taping was applied to the chest and lumbar regions, while in the control group, the placement was not done in the indicated way. The measurements were performed two days before and two days after the intervention. There was a significant increase in the Berg Balance Scale (BBS) $(p<.001)$ and in the Timed Up-andGo (TUG) test $(p<.001)$ shortly after taping and after its removal. The study confirmed the efficacy of the method in the dynamic balance and mobility of children with $\mathrm{CP}$ and can potentially be used in addition to neurorehabilitation.
In another study, Özmen et al. ${ }^{[11]}$ in a sample of 19 children with $\mathrm{CP}$, investigated the effect of $\mathrm{KT}$ on balance and gait preparation. A goniometer measured the range of motion of the ankle from the supine position. Muscle tone in plantar flexion and gait preparation was measured with the Ashworth scale, the One-minute walk test and the TUG test. No difference was seen during the first 48 hours. However, there were significant differences in measurements after this period $(p<.05)$, as balance and gait preparation improved. Ibrahim ${ }^{[12]}$ studied the effect of KT on 30 children with $\mathrm{CP}$ to draw conclusions about its effect on trunk control and balance. In the intervention that lasted 12 weeks, two groups with an equal number of children were created. Taping was applied in the intervention group, while in the control group only classical physiotherapy was used. Measurements were made before and after treatment. It appeared that trunk control in the sitting and standing positions and balance improved significantly in both groups. However, statistically the benefits were greater in the intervention group, except for pelvic rotation which was not statistically different between the two groups.

The improvement of functioning with the use of KT was the research focus in the study of Kaya Kara et al. ${ }^{[13]}$. The study involved 30 children with $\mathrm{CP}$, who were divided equally between the intervention group, in which KT was applied, and the control group, in which physiotherapy was applied, for 12 weeks. The statistical analysis showed a significant difference in terms of muscle strength $(p=.003)$, lateral gait $(p=.016)$ and standing up $(p=.018)$ between the two groups $(p<.05)$. Intervention with taping in these patients can have positive effects in terms of increasing proprioception and better functioning in their daily lives.

Simsek et al. ${ }^{[14]}$, in a sample of 31 in a sample of 31 children with $\mathrm{CP}$, studied the results of the application of $\mathrm{KT}$ in the sitting position regarding the motor function and the functional autonomy of the individuals in general. The program had a duration of 12 weeks and the children were divided into two groups. The intervention group was treated with KT and the control group with kinesiotherapy. The results showed that there was an improvement in the intervention group compared to the control group in terms of motor function in the sitting position of children with CP. This improvement, however, did not become immediately apparent after the intervention. The application of taping can be beneficial as a complementary method in combination with physiotherapy.

In the study of Dixit et al. ${ }^{[15]}, 60$ children with $\mathrm{CP}$ were studied in regards to the benefits of KT in terms of mobility and function in the femoral muscles. The children were divided into two groups. In the intervention group, occupational therapy and KT techniques were applied to the knee flexors and extensors, while in the control group only occupational therapy was applied. The results showed that after the application the muscles in the intervention group were stronger, as well as the mobility of the children.

Lastly, Da Costa et al. ${ }^{[16]}$, in a sample of four children with $\mathrm{CP}$, investigated the efficacy of KT in relation to sitting-up movement, balance and dynamic postural control. The measurements were made with and without KT application and for the analysis the TUG test, PSB Dynamic activities and transitions from sitting to standing position were used. The results showed benefits in terms of dynamic activities, such as reducing the time of moving from sitting to standing with less ankle flexion and greater knee extension, as well 
as in the TUG test. There were no benefits for static activities such as balance (PSB) with the application of KT.

\section{Discussion-Conclusions}

To combat the serious effects of $\mathrm{CP}$ on mobility and ultimately on the functioning of children, there are many techniques and methods used by physiotherapists. KSome of them are classic and tried and tested methods, such as Bobath, Vojta, PNF and Katona, while in recent years the combination-selective treatment that aims at a combination of methods and not monotherapy is gaining ground ${ }^{[1]}$. One of the modern treatments in general and in particular is the application of KT, which in combination with other approaches is chosen to be used in this category of patients. In this literature review, it was shown that the application of $\mathrm{KT}$ in children with $\mathrm{CP}$ has positive effects regarding the increase of the function of the trunk and lower limbs. In most cases, it turns out that this happens not immediately after application, but shortly after its removal ${ }^{[7,10,11,14]}$. In addition, the combination of KT together with other physiotherapy interventions seemed to lead to even better results ${ }^{[12-14]}$, although more studies will be needed to draw safer conclusions.

\section{References}

1. Paras G. Methods of Physiotherapeutic approach in children with cerebral palsy: Myth and reality. Physiother issues 2006;4(2):5-14.

2. Ziebell M, Imms C, Froude EH, McCoy A, Galea M. The relationship between physical performance and self-perception in children with and without cerebral palsy. Aust Occup Ther J 2009;56(1):24-32.

3. Kosmetatou E. Cerebral palsied children. Panteion 2010.

4. Dollani Dimitra AM. Gait of children with diplegic cerebral palsy. Technological Educational Institute of Western Greece 2015.

5. Das Sakti P, Ganesh GS. Evidence-based Approach to Physical Therapy in Cerebral Palsy. Indian J Orthop 2019;53(1):20-34.

6. Psarianou E. Physiotherapeutic approach in children with dyskinetic cerebral palsy. Technological Educational Institute of Western Greece 2018.

7. Tabatabaee M, Cheraghifard M, Shamsoddini A. The effects of kinesio taping of lower limbs on functional mobility, spasticity, and range of motion of children with spastic cerebral palsy. Egypt J Neurol Psychiatry Neurosurg 2019;55(1):1-6.

8. Jung S-H, Song S-H, Kim D-R, Kim S-G, Park Y-J, Son Y-J et al. Effects of kinesio taping on the gait parameters of children with cerebral palsy: a pilot study. Phys Ther Rehabil Sci 2016;5(4):205-9.

9. Dos Santos AN, Visicatto LP, de Oliveira AB, Rocha NACF. Effects of Kinesio taping in rectus femoris activity and sit-to-stand movement in children with unilateral cerebral palsy: placebo-controlled, repeatedmeasure design. Disabil Rehabil [Internet] 2019;41(17):2049-59. Available from: https://doi.org/10.1080/09638288.2018.1458912

10. Partoazar B, Ganji B, Dalvand H, Shamsoddini A. The effect of trunk kinesio taping on static and dynamic balance and functional mobility in children with cerebral palsy. Sport Sci Health [Internet] $\begin{array}{lcl}2021 ; 17(1): 111-7 . \quad \text { Available } & \text { from: } \\ \text { https://doi.org/10.1007/s11332-020-00660-4 } & \end{array}$

11. Özmen T, Acar E, Zoroğlu T, Işik H. Effect of kinesio taping on gait performance and balance in children with hemiplegic cerebral palsy. Fiz Rehabil 2017;28(1):33-7.

12. Ibrahim MM. Investigating the Effect of Therapeutic Taping on Trunk Posture and Control in Cerebral Palsy Children with Spastic Diplegia. J Med Sci Clin Res 2015;03(09):7452-9.

13. Kaya Kara O, Atasavun Uysal S, Turker D, Karayazgan S, Gunel MK, Baltaci G. The effects of Kinesio Taping on body functions and activity in unilateral spastic cerebral palsy: A single-blind randomized controlled trial. Dev Med Child Neurol 2015;57(1):81-8.

14. Şimşek TT, Türkücüoğlu B, Çokal N, Üstünbaş G, Şimşek IE. The effects of Kinesio ${ }^{\circledR}$ taping on sitting posture, functional independence and gross motor function in children with cerebral palsy. Disabil Rehabil 2011;33(21-22):2058-63.

15. Dixit J. Effect of Kinesiotape in Improving Mobility Capacity in Children with Cerebral Palsy. J Med Sci Clin Res 2018;6(8):1257-64.

16. Da Costa CSN, Rodrigues FS, Leal FM, Rocha NACF. Pilot study: Investigating the effects of Kinesio Taping ${ }^{\circledR}$ on functional activities in children with cerebral palsy. Dev Neurorehabil 2013;16(2):121-8. 